\title{
Generalized Quantum Theory: Overview and Latest Developments
}

\author{
Thomas Filk • Hartmann Römer
}

Received: 17 September 2010/Accepted: 27 October 2010/Published online: 13 November 2010

(C) Springer Science+Business Media B.V. 2010

\begin{abstract}
The main formal structures of generalized quantum theory are summarized. Recent progress has sharpened some of the concepts, in particular the notion of an observable, the action of an observable on states (putting more emphasis on the role of proposition observables), and the concept of generalized entanglement. Furthermore, the active role of the observer in the structure of observables and the partitioning of systems is emphasized.
\end{abstract}

Keywords Generalized quantum theory · Complementarity · Entanglement

\section{Introduction}

The motivation for the formulation of Generalised Quantum Theory (GQT) (Atmanspacher et al. 2002, 2006), whose minimal version will be presented here, was the desire to sharpen the often vague and metaphoric usage of originally quantum theoretical terms like complementarity and entanglement in fields of knowledge differing from physics. Starting from the algebraic formulation of physical quantum theory and discarding from its axioms elements appearing to be special to physics, a formalism was obtained which is applicable far beyond the realm of physics and still rich enough to attribute a well defined formal meaning to the notions of complementarity and entanglement beyond physics. Meanwhile, quite a few applications demonstrating a partial structural isomorphy to quantum physics in rather diverse situations have been worked out in more or less detail (see Atmanspacher et al. 2002, 2004, 2006, 2008a, b; Römer 2004, 2006, 2007, 2008;

T. Filk $(\bowtie) \cdot$ H. Römer

Department of Physics, University of Freiburg, Hermann-Herder-Str. 3, 79104 Freiburg, Germany

e-mail: thomas.filk@physik.uni-freiburg.de

T. Filk

Parmenides Foundation for the Study of Thinking, Munich, Germany 
Römer and Walach 2008; Walach and Römer 2009; von Lucadou et al. 2007; von Lucadou and Römer 2010; Walach 2002, 2005).

The formalism of GQT was called "weak quantum theory" in the original publication (Atmanspacher et al. 2002) because it arose by weakening the axioms of physical quantum theory. However, the wider range of applicability of this theory makes its designation as "weak" inappropriate, and for this reason we now use the term "generalized quantum theory". In this short contribution we will sketch a slight reformulation, simplification and further generalisation of GQT. This new formulation puts stronger emphasis on the operationalisable features of GQT and leaves intact all its applications, in particular the formally precise formulation of complementarity and entanglement. Details will be given in a more comprehensive publication (Atmanspacher et al., in preparation).

The reader who is not so familiar with the algebraic concepts and the mathematical formalism of operators, propositions, eigenvectors etc. may leave aside the mathematical aspects and formulae and stick to the explanatory remarks.

\section{The Formalism of GQT}

Generalized quantum theory takes over from both classical and quantum physics the following four notions:

System: A system is anything which can be (imagined to be) isolated from the rest of the world and be subject to an investigation. A system can be as general as an object or a school of art together with all persons involved in production and interpretation. Unlike the situation in, e.g., classical mechanics the identification of a system is not always a trivial procedure but sometimes a creative act, in particular in view of the possibility of entanglement with other systems to be described in Sect. 4 In many cases it is possible to define subsystems inside a system

State: A system must have the capacity to reside in different states without losing its identity as a system. One may differentiate between pure states, which correspond to maximal possible knowledge of the system and mixed states corresponding to incomplete knowledge. Notice that the notion of a mixed state contains an epistemic element. We shall see later that this also applies to some extent to pure states.

Observable: An observable corresponds to any feature of a system, which can be investigated in a more or less meaningful way. Global observables pertain to the system as a whole, local observables pertain to subsystems. (For details concerning the subjective element of partitioning and identifying subsystems, see Sect. 4) Observables may, for instance, correspond to esthetic investigations for systems of art (schools).

Measurement: Doing a measurement of an observable $A$ means performing the investigation which belongs to the observable $A$ and arriving at a result $a$, which can claim factual validity. What factual validity means depends on the system: Validity of a measurement result for a system of physics, internal conviction for self observation, consensus for groups of human beings. The result of the measurement 
of $A$ will in general depend on the state $z$ of the system before measurement but will not be completely determined by it.

To every observable $A$ we associate its spectrum, a set Spec $A$, which is just the set of all possible measurement results of $A$. Immediately after a measurement of an observable $A$ with result $a$ in $\operatorname{Spec} A$, the system will be in an eigenstate $z_{a}$ of the observable $A$ with eigenvalue $a$. The eigenstate $z_{a}$ is a state, for which an immediate repetition of the measurement of the same observable $A$ will again yield the same result $a$ with certainty, and after this repeated measurement the system will still be in the same state $z_{a}$. This property which is also crucial in quantum physics justifies the terminology "eigenstate of an observable $A$ " for $z_{a}$ and "eigenvalue" for the result $a$. (We should notice that this does not correspond to a mathematical eigenvalue equation, however, the relation to the mathematical notion of eigenstate in quantum physics will be given in Sect. 3.) We emphasize that this is an idealized description of a measurement process.

Even in quantum physics, the attempts to describe the physical measuring process entirely within its own formalism have not been fully successful so far (Bell 2004). GQT deals with systems, whose physical character is not focal and often unessential. Generalised "measurement" in GQT is predominantly a cognitive process, though, of course, with a physical substrate.

Two observables $A$ and $B$ are called complementary, if the corresponding measurements are not interchangeable. This means that the state of the system depends on the order in which the measurement results, say $a$ and $b$, were obtained. If the last measurement was a measurement of $A$, the system will end up in an eigenstate $z_{a}$ of $A$, and if the last measurement was a measurement of $B$, an eigenstate $z_{b}$ will result eventually. For complementary observables $A$ and $B$ there will be at least some eigenvalue, say $a$, of one of the observables for which no common eigenstate $z_{a b}$ of both observables exists. This means that it is not generally possible to ascribe sharp values to the complementary observables $A$ and $B$, although both of them are equally important for the description of the system. This is the essence of quantum theoretical complementarity which is well defined also for GQT. Sometimes, a stronger notion of complementarity is employed, which is motivated by the quantum mechanical complementarity between the position observable $Q$ and the momentum observable $P$. Here, any definite value of one of these observables means complete uncertainty of the measurement value of the other observable. This more restrictive notion of complementarity can be taken over to a large extent to GQT: We call the observables $A$ and $B$ strongly complementary, if there exists no common eigenstate for both of them. Strongly complementary observables are, of course, complementary.

Non complementary observables, for which the order of measurement does not matter, are called compatible. After the measurement of compatible observables $A$ and $B$ with results $a$ and $b$, the system will be in the same common eigenstate $z_{a b}$ of $A$ and $B$ irrespective of the order in which the measurements were performed.

Propositions are special observables corresponding to "yes-no" questions to the system [for the general formalism of quantum mechanics and propositions, see any text book on quantum theory, e.g. Thirring (2002)]. Thus the spectrum Spec $P$ of a proposition $P$ is contained in the set $\{$ yes, no $\}$. We define trivial propositions $\mathbf{1}$ and $\mathbf{0}$ such that $\mathbf{1}$ is always true (the result is always "yes") and $\mathbf{0}$ is never true. 
Furthermore, to every proposition $P$ we associate is negation $\neg P$, which returns the measurement value "yes" if $P$ returns "no" and returns "no" if $P$ returns "yes". Evidently, $\neg(\neg P)=P, P$ and $\neg P$ are compatible, $\neg \mathbf{1}=\mathbf{0}$. Furthermore, $\mathbf{1}$ and $\mathbf{0}$ are compatible with all observables.

For compatible (and in fact only for compatible) propositions $P$ and $Q$ we define a conjunction $P$ AND $Q$, which gives the measurement value "yes" if and only if both $P$ and $Q$ give "yes" and an adjunction $P$ OR $Q=\neg(\neg P$ AND $\neg Q)$, which yields "yes" if and only if $P$ or $Q$ yield "yes". $P$ and $Q$ are compatible with $P$ AND $Q$ and $P$ OR $Q$. Simple identities like

$$
1 \text { AND } P=P \text { and } P \text { AND } \neg P=\mathbf{0}
$$

hold. For details see the original reference (Atmanspacher et al. 2002).

In physical quantum theory states are represented by non-negative hermitean density matrices $z \neq o$ with $z \geq 0$ and $z^{+}=z$. A state $z$ then associates to an observable $A$ its expectation value $z(A)=\operatorname{tr}(z A) / \operatorname{tr}(z)$. (So, for a number $c \geq 0, z$ and $c z$ define the same expectation value function.) Observables in physical quantum theory can be identified with mappings, which to every state $z$ associate another state $A(z)=A z A$. However, $A(z)$ may be the zero matrix, and the price be paid for defining an action of observables on states is the necessity to admit an additional improper zero state $o$.

In GQT, states are in general not given by density matrices but one can still define an action of proposition observables on states after admitting an improper zero state $o$. Using the structure obtained so far, one defines for any proposition $P$

- $P(o)=o$, and for $z \neq o$

- $P(z)=o$, if the measurement result "yes" for $P$ is impossible in the state $z$

- $P(z)=$ the state obtained after a measurement of $P$ with result "yes", if the result "yes" is possible in the state $z$.

This definition imitates the action of propositions on states in quantum physics. The action of propositions $P$ on states employs measurements of $P$ and is operationally well defined.

We immediately see

$$
\mathbf{0}(z)=o, \quad \mathbf{1}(z)=z
$$

Moreover, for (compatible or complementary) propositions $P$ and $Q$ we can define a composition $P Q$ as mappings on states by

$$
P Q(z)=P(Q(z))
$$

and we have

$$
\begin{aligned}
& P P(z)=P(z), \quad \text { i.e. } \quad P P=P \\
& P \neg P=\neg P P=0 \\
& \mathbf{0} P=P \mathbf{0}=\mathbf{0}, \quad \mathbf{1} P=P \mathbf{1}=P
\end{aligned}
$$

A proposition $P$ can be identified with the pair of mappings on states associated to $P$ and $\neg P$, and two propositions $P$ and $Q$ are compatible, if and only if all the propositions $P, \neg P, Q, \neg Q$ commute as mappings on states, i. e., if and only if 


$$
P Q=Q P, \quad P \neg Q=\neg Q P, \quad \neg P Q=Q \neg P, \quad \neg P \neg Q=\neg Q \neg P .
$$

For compatible propositions $P$ and $Q$

$$
P \text { AND } Q=P Q=Q P
$$

holds.

Quite generally, observables can be reduced to families of propositions in the following way: For every $a$ in $\operatorname{Spec} A$ let $A_{a}$ denote the proposition corresponding to the assertion that the value of $A$ is $a$. In particular, for a proposition $P$ we have

$$
P_{\text {yes }}=P \quad \text { and } \quad P_{\text {no }}=\neg P .
$$

Evidently

$$
A_{a} A_{a^{\prime}}=\mathbf{0} \quad \text { for } a \neq a^{\prime}
$$

and

$$
\bigcup_{a \in \mathrm{SpecA}} A_{a}=\mathbf{1}
$$

This simply means that the propositions $A_{a}$ are mutually exclusive and that after a measurement exactly one of them has to be true.

In fact an alternative way to build up the formalism of GQT would be to start out from propositions and to define observables $A$ as pairs $\left(\operatorname{Spec} A,\left(A_{a}\right)_{a \in \operatorname{SpecA}}\right)$ consisting of the set Spec $A$ and a family of propositions $A_{a}$ with the properties defined by the last two formulae.

The observable $A$ and the propositions $A_{a}$ are compatible and two observables $A$ and $B$ are compatible if and only if all the propositions $A_{a}$ and $B_{b}$ are compatible.

In the original formulation of GQT, like in physical quantum theory, the existence of an action of observables on states was postulated. Even in physical quantum theory this action is operationally not easily accessible. In our new formulation we restrict ourselves to the introduction of an operationally better defined action of propositions on states. This simplification and further weakening of the axioms of GQT has no affect on the definition of a generalized notion of complementarity and, as we shall see, entanglement.

In concrete applications of GQT, however, it may be advantageous to go beyond the minimal formal scheme of GQT and use an action of all observables on states. This is for instance the case for the application of GQT to bistable perception quoted in Atmanspacher et al. (2004).

\section{Comments on Some Aspects of GQT}

Comparing physical quantum theory with the (original or new formulation of) GQT one readily finds the following essential differences:

- In GQT there is no quantity like Planck's constant controlling the degree of complementarity of observables. Thus, contrary to physical quantum theory, 
where quantum effects are essentially restricted to the microscopic regime, macrosopic quantum like effects in GQT are to be expected.

- At least in its minimal version described here, GQT contains no reference to time or dynamics.

- In its minimal version GQT does not ascribe quantified probabilities to the outcomes of measurements of an observable $A$ in a given state $z$. Indeed, to give just one example, for esthetic observables quantified probabilities seem to be inappropriate from the outset. What rather remains are modal logical qualifications like "impossible", "possible" and "certain". Related to the absence of quantified observables, the set of states in GQT is in general not modeled by a linear Hilbert space. Moreover, no addition of observables (operationally difficult to access even in quantum physics) is defined in GQT.

- In quantum physics where states are defined by means of a linear Hilbert space observables can be identified with linear maps of the Hilbert space into itself. Furthermore, an eigenstate of an observable $A$ is a state which is reproduced by the linear action of observables on states. In GQT only proposition observables act on states, as mentioned above. Moreover, linearity of this action cannot be defined. The salient feature of an eigenstate in quantum mechanics is that further measurements of the same observable without intervening disturbations will reproduce it. This decisive and operationally well-defined feature is assumed in our definition of an eigenstate in GQT.

- Related to this, GQT in its minimal form provides no basis for the derivation of inequalities of Bell's type for measurement probabilities, which allow for the conclusion, that the indeterminacies of measurement values are of an intrinsic nature rather than a lack of knowledge. It is an open question whether the construction of states analogous to GHZ-states in quantum physics (Greenberger et al. 1989, 1990), which allow for a decision in favour of ontic indeterminacies by one single measurement, is possible in GQT. As such, GQT is a phenomenological theory and leaves the question for the ontic or epistemic nature of indeterminacies open. The answer may depend on the concrete system considered. After all, physical quantum theory with ontic indeterminacies is a special case of GQT. In many applications of GQT indeterminacies may be epistemic and due to incomplete knowledge of the full state or uncontrollable perturbations by outside influences or by the process of measurement. Notice that complementarity in the sense of GQT may even occur in coarse grained classical dynamical systems (beim Graben and Atmanspacher 2006, 2008).

- For some applications (see e.g., Atmanspacher et al. 2004, 2008a, b) one may want to enrich the above described minimal scheme of GQT by adding further structure, e.g., an underlying Hilbert space structure for the states.

We should stress here that for very general systems like schools of art, observables are not so directly given by the system and read off from it like location and velocity in a mechanical system. On the contrary, as already suggested by the name of an "observable", the identification of an observable may be a highly creative act of the observer, which will be essentially determined by his horizon of questions and expectations. This marks a decidedly epistemic trait of the notion of 
observables in GQT even more than in quantum physics. Moreover, the horizon of the observer will change, not the least as a result of his previous observations adding to the open and dynamical character of the set of observables.

\section{Partitions and Entanglement}

What has just been said about observables also applies to partitioning a system into subsystems. In fact, partitioning is achieved by means of partition observables whose different values differentiate between the subsystems. In general, subsystems do not preexist in a naïve way but are in a sense created in the constitutive act of their identification.

Different partitions may be compatible or complementary. The physical position observable $Q$ is a privileged example of a partition observable defining a partition into spatially separated subsystems. The range of applicability of this partition observables is largely coincident with the range of physics (physics as the realm of "res extensae").

The first partition prior to every further one and prerequisite for any act of cognition is the epistemic split into observer and observed system: After all, any cognition of the kind we have access to is the cognition of someone about something. One might speculate about the existence of splittings which are complementary to any epistemic split.

In quantum physics, the epistemic split is known under the name of Heisenberg split. It can be moved and shifted but it is inevitable for any measurement. In the quantum theory of physical measurement one can observe that the stochasticity of measurement results enters as a result of the Heisenberg split and subsequent projection onto either the subsystem of the observer or the observed object. There is a symmetry in the probabilities of measured values with respect to projection onto measured system and measuring device. One might ask oneself, to what extent this symmetry could be generalized in GQT.

The genuinely quantum theoretical phenomenon of entanglement can and in general will show up also in GQT if the following conditions are fulfilled:

1. A system is given for which subsystems can be identified. Entanglement phenomena will be best visible if the subsystems are sufficiently separated. Local observables pertaining to different subsystems are compatible.

2. There is a global observable of the total system, which is complementary to the local observables of the subsystems.

3. Given these two prerequisites, a system will be in an entangled state if this state is an eigenstate of the above mentioned global observable and not an eigenstate of the local observables. (In GQT this is the definition of an entangled state, while in quantum theory this can be shown to be equivalent to the usual definition of an entangled state as not being separable; see e.g., (Nielsen and Chuang 2000), which in general is a good reference for all questions concerning entanglement in quantum physics.) 
Fig. 1 Entanglement

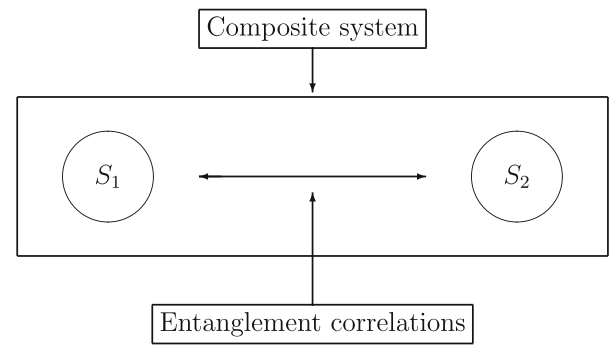

Given these conditions, the measured values of the local observables will be uncertain because of the complementarity of the global and the local observables. However, so-called entanglement correlations will be observed between the measured values of the local observables (see Fig. 1). These correlations are non local and instantaneous. Einstein, trying to argue for an incompleteness of quantum mechanics, spoke about "spooky interactions" in this connection. Entanglement correlations have been observed beyond any doubt in quantum physics (see Nielsen and Chuang 2000 and references therein). Entanglement correlations are not due to causal interactions between the subsystems.

Such correlations without interactions are a witness of the holistic character of composite quantum systems: In general, the states of the subsystems do not determine the state of the total system. Vice versa, the holistic state of the total system does not determine the measured values of local observables pertaining to the subsystems. The holistic character of the total quantum state resides in entanglement correlations between the subsystems.

It is not difficult to show that in quantum physics entanglement correlations cannot be used for signal transmission between different subsystems. This last statement is sometimes referred to as Eberhards theorem (Eberhard 1978). For a simple explanation see von Lucadou et al. (2007). This must also hold in GQT in order to prevent bizarre intervention paradoxes. One may even turn the argument around and state that, whenever correlations between subsystems can be used for signal transfer, they must be of causal nature and entanglement must be absent or at least not dominant.

For completeness, we should mention here, that a distinction between local and global observables is also possible for temporal separations (see, for instance Atmanspacher et al. 2008).

As already mentioned above, in quantum physics inequalities for entanglement correlations of Bell's type can be employed to show that the indeterminacies of the measured values of local observables, and, more generally, of any quantum observable, can be of ontic rather than epistemic nature. Bell's inequalities also allow to differentiate between a genuine entangled state and a mixture of product states, which will show similar correlations between subsystems.

In GQT in its minimal form there are no Bell inequalities and the above differentiation may be difficult or impossible. In such situations, GQT should be considered a phenomenological theory which leaves the question for the ontic or epistemic character of indeterminacies open. 
We already saw that observables in quantum theory, and even more so in GQT, are not exclusively to be attributed to the observed system but have a strong reference to the observer. They are to be located on the epistemic split and hence have a certain epistemic connotation.

This also applies to the notion of a state. In classical mechanics, a pure state can be interpreted as an entirely ontic entity. In quantum physics and certainly also in GQT, the notion of a state is not so much related to "what there is" in the system but rather to what will be observed. This gives a definite epistemic colouring even to the predominantly ontic notion of a pure state, which applies in particular if GQT is interpreted as a phenomenological theory of systems.

Acknowledgments Both authors acknowledge numerous discussions with Harald Atmanspacher. Part of this project was financed by the Fetzer-Franklin Fund.

\section{References}

Atmanspacher H, Römer H, Walach H (2002) Weak quantum theory: complementarity and entanglement in physics and beyond. Found Phys 32:379-406

Atmanspacher H, Filk T, Römer H (2004) Quantum Zeno features of bistable perception. Biol Cybern 90:33-40

Atmanspacher H, Filk T, Römer H (2006) Weak quantum theory: formal framework and selected applications. In: Adenier G, Khrennikov AYu, Nieuwenhuizen TM (eds) Quantum theory: reconsiderations of foundations. American Institute of Physics, New York, pp 34-46

Atmanspacher H, Bach M, Filk T, Kornmeier J, Römer H (2008a) Cognitive time scales in a Necker-Zeno model for bistable perception. Open Cybern Syst J 2:234-251

Atmanspacher H, Filk T, Römer H (2008b) Complementarity and bistable perception. In: Atmanspacher H, Primas H (eds) Recasting reality, Wolfgang Pauli's philosophical ideas and contemporary science. Springer. ISBN 978-3-540-85197-4

Atmanspacher H, Filk T, Römer H (in preparation)

beim Graben P, Atmanspacher H (2006) Complementarity in classical dynamical systems. Found Phys 36:291-306

beim Graben P, Atmanspacher H (2008) Extending the philosophical significance of the idea of complementarity. In: Atmanspacher H, Primas H (eds) Recasting reality, Wolfgang Pauli's philosophical ideas and contemporary science. Springer

Bell J (2004) Against measurement; in Speakable and unspeakable in quantum mechanics, 2nd edn. University of Cambridge Press, Cambridge

Eberhard P (1978) Bell's theorem and the different concepts of locality. Nuovo Cimento 46B:392-419

Greenberger DM, Horne M, Zeilinger A (1989) Going beyond Bell's theorem. In: Kafatos M (eds) Bell's theorem, quantum theory, and conceptions of the universe. Kluwer, Dordrecht, p 69

Greenberger DM, Horne MA, Shimony A, Zeilinger A (1990) Bell's theorem without inequalities. Am J Phys 58:1131

Nielsen MA, Chuang IL (2000) Quantum computation and quantum information. Cambridge University Press, Cambridge

Römer H (2004) Weak quantum theory and the emergence of time. Mind Matter 2:105-125

Römer H (2006) Complementarity of process and substance. Mind Matter 4:69-89

Römer H (2007) Konsistente und inkonsistente Geschichten. In Zeitschrift für Parapsychologie und Grenzgebiete der Psychologie (preprint)

Römer H (2008) Verschränkung. In: Knaup M, Spät P (eds) Post-Physikalismus. Verlag Karl Alber, Freiburg (to appear)

Römer H, Walach H (2008) Complementarity between phenomenal and physiological observables (preprint)

Thirring W (2002) Quantum mathematical physics: atoms, molecules and large systems. Springer, Berlin 
von Lucadou W, Römer H (2010) Schuld Person und Gesellschaft: Systemische Perspektiven. In: The proceedings of the 10. Mannheimer Ethik-Symposium Schuld: Bearbeitung, Bewältigung, Lösung, Interdisziplinäre gesellschaftliche und psychotherapeutische Perspektiven, Jan 2010 (to appear)

von Lucadou W, Römer H, Walach H (2007) Synchronistic phenomena as entanglement correlations in generalized quantum theory. J Conscious Stud 14:50-74

Walach H (2002) Entanglement model of homeopathy as an example of generalised entanglement predicted by weak quantum theory. Forschende Komplementärmedizin und klassische Naturheilkunde 10:192-20

Walach H (2005) Research on homeopathy: state of the art. J Altern Complement Med 5:813-829 (and references therein)

Walach H, Römer H (2009) Generalised entanglement—a nonreductive option for a phenomenologically dualist and ontologically monist view 